\title{
A population synthesis study on the faint X-ray sources in the Galactic center region
}

\author{
X.-W. Liu and X.-D. Li
}

Department of Astronomy, Nanjing University, Nanjing 210093, PR China

e-mail: [liuxw;lixd]@nju.edu.cn

Received 29 March 2005 / Accepted 10 November 2005

\section{ABSTRACT}

Context. Recent Chandra observations of the Galactic center region (GCR) have uncovered a population of faint discrete X-ray sources. A few theoretical works have already investigated the nature of these sources.

Aims. We examine the contributions and luminosity functions of various kinds of candidate objects that have been proposed either by previous authors or by ourselves.

Methods. We conduct a population synthesis calculation based on the Hurley et al. rapid binary evolution code. Several candidate models, i.e. wind-accreting neutron stars, intermediate polars, low mass X-ray binaries, young pulsars, and massive stars with strong winds, are incorporated into our calculation. We also take the geometric effect of the accretion disk into account for Roche lobe overflow X-ray binaries. Results. Our results show that neutron star low-mass X-ray binaries contribute significantly to the observed sources. We also point out that wind-accreting neutron stars contribute negligible amount to these sources due to the propeller effect, and the intermediate polars play a minor role in accounting for the faint X-ray sources in both the Wang et al. and Muno et al. survey. It should be mentioned that the majority of the sources in the survey field of Wang et al. are still beyond what we can expect to find.

Key words. stars: evolution - stars: neutron - stars: binaries: close - X-rays: binaries - methods: statistical

\section{Introduction}

Instrumental development always leads investigators to look for fainter and fainter sources to study. Recent Chandra observations of the Galactic center region (GCR) have uncovered a population of faint discrete X-ray sources. Wang et al. (2002) have reported hundreds of X-ray sources with luminosities $L_{\mathrm{X}} \sim 10^{33}-10^{35} \mathrm{erg} \mathrm{s}^{-1}$ in the GCR, which contains $\lessgtr 1 \%$ of the total Galactic population (Pfahl et al. 2002, hereafter PRP02). Many of these sources are emitting in a hard energy $(>3 \mathrm{keV})$ band since the $\mathrm{X}$-ray absorption, varying across the field, is particularly high in the $1-3 \mathrm{keV}$ band. Muno et al. (2003) presents a catalog of 2357 point sources detected during their 590 ks Chandra observations of the $17^{\prime} \times 17^{\prime}$ field around Sgr A*. It was estimated that there are $\sim 2000$ individual pointlike sources with luminosities $L_{\mathrm{X}} \sim 10^{30}-10^{33} \mathrm{erg} \mathrm{s}^{-1}$ lying within a cylinder of radius $20 \mathrm{pc}$ and depth $440 \mathrm{pc}$ centered on the Galactic center at a distance of $8.5 \mathrm{kpc}$ (Muno et al. 2004a). The spectra of these sources can be fitted by an absorbed power law with photon index $\Gamma$. More than 1000 sources have relatively hard spectra with $\Gamma<1$.

The nature of the faint X-ray sources in the GCR has been investigated by several authors. Pfahl et al. (2002) attribute a significant fraction of the X-ray sources revealed by
Wang et al. (2002) to wind-accreting neutron stars (WNSs). By calculating the flux distribution of WNSs, they conclude that the detected number ranges from ten to several hundred. Similar idea for pre-low-mass X-ray binaries is suggested by Willems \& Kolb (2003; see however, Popov 2004). Muno et al. (2004a) propose that magnetized cataclysmic variables (CVs), referred to as polars and intermediate polars (IPs), could account for $\sim 1000-2000 \mathrm{X}$-ray sources in their survey. The population synthesis work by Belczynski \& Taam (2004, hereafter BT04) suggests that transient neutron star low-mass X-ray binaries (NS LMXBs) may contribute primarily to the X-ray source population in the Muno et al. survey.

In this paper, we employ the evolutionary population synthesis (EPS) method to calculate the expected numbers and luminosity distributions of various types of candidates that emit X-rays in the Wang et al. and Muno et al. surveys. We examined the spin evolution of WNSs proposed by PRP02, including the ejector, propeller, and accretor stages (Davies \& Pringle 1981, hereafter DP81; Lipunov 1992; Ikhsanov 2001, 2002). Besides traditional X-ray binaries and CVs, we also considered the contribution from rotation-powered pulsars and massive stars with strong winds. We describe the population synthesis method and the input physics for various types of X-ray sources in our model in Sect. 2. The calculated results are 
presented in Sect. 3. Our discussions and conclusions are found in Sect. 4.

\section{Model description}

We use the EPS code developed by Hurley et al. (2000, 2002) to calculate the expected numbers in the Galactic disk for various types of single and binary X-ray source populations. This code incorporates the evolution of single stars with binary-star interactions, such as mass transfer, mass accretion, commonenvelope (CE) evolution, collisions, supernova kicks, tidal friction, and angular momentum loss mechanics. Most of our adopted parameters are the same as those described in Hurley et al. (2002). We assume all stars are born in binary systems. The initial mass function (IMF) of Kroupa et al. (1993) is taken for the primary's mass $\left(M_{1}\right)$ distribution (discussed below in Sect. 2.3). For the secondary stars $\left(M_{2}\right)$, we assume a uniform distribution of the mass ratio $M_{2} / M_{1}$ between 0 and 1 . A uniform distribution of $\ln a$ is also taken for the binary separation $a$. The star formation rate parameter is $S=7.6085 \mathrm{yr}^{-1}$, corresponding to a rate of $\sim 0.02 \mathrm{yr}^{-1}$ for core-collapse supernovae ( $\mathrm{SNe}$ ) in our Galaxy, assuming all the stars with masses $>8 M_{\odot}$ die through $\mathrm{SNe}$. During the SN explosions, a kick velocity $v_{\mathrm{k}}$ is imparted on the newborn compact stars with the Maxwellian distribution

$P\left(v_{\mathrm{k}}\right)=\sqrt{\frac{2}{\pi}} \frac{v_{\mathrm{k}}^{2}}{\sigma^{3}} \exp \left(-\frac{v_{\mathrm{k}}^{2}}{2 \sigma^{2}}\right)$

where $\sigma=265 \mathrm{~km} \mathrm{~s}^{-1}$ (Hobbs et al. 2005) or $190 \mathrm{~km} \mathrm{~s}^{-1}$ (Hansen \& Phinney 1997). The CE parameter $\alpha_{\mathrm{CE}}$ is set to be 1 , if not otherwise mentioned. The velocity $v_{\mathrm{W}}$ of winds from massive stars is taken to be $v_{\mathrm{W}}=\sqrt{2 \beta(G M / R)}$, where the value of $\beta$ depends on the spectral type of the stars. Hurley et al. (2002) suggests it is in the range of 0.125-7.0.

\subsection{Physical models for $X$-ray sources}

\subsubsection{WNSs}

As in PRP02, in our model a WNS is a neutron star (NS) accreting wind material from an intermediate- or high-mass $\left(>3 M_{\odot}\right)$ un-evolved companion. We assume that the wind is steady and spherically symmetric. The standard Bondi-Hoyle (1944) accretion formula is employed to calculate the mass transfer rate to the NS. The Be/NS binaries are not considered in the present model, due to their high eccentricities and the unclear structure of the Be star's equatorial winds. Calculations in PRP02 simply assume that a WNS always accretes from the wind of its binary companion, ignoring its previous evolution as an ejector and propeller. Obviously the expected number of WNSs would be less than in PRP02, depending on the duration of the latter stages. The following equations give the estimates of the timescales for the ejector (a), supersonic (c), and subsonic (d) propeller phases for a natal NS in a wind environment (DP81; Ikhsanov 2002),

$$
\begin{aligned}
& \tau_{a} \simeq 4.8 \times 10^{6} \mu_{31}^{-1} \dot{M}_{17}^{-1 / 2} I_{45} V_{7}^{-1} \mathrm{yr}, \\
& \tau_{c} \simeq 10^{6} \mu_{31}^{-1} \dot{M}_{17}^{-1 / 2} I_{45} V_{7}^{-1} \mathrm{yr},
\end{aligned}
$$

$\tau_{d} \simeq 10^{3} \mu_{31}^{-2} m I_{45} P_{100 \mathrm{yr},}$

where $\mu=10^{31} \mu_{31} \mathrm{G} \mathrm{cm}^{3}$ is the magnetic moment, $P=$ $100 P_{100} \mathrm{~s}$ the spin period, $M_{\mathrm{NS}}=m M_{\odot}$ the mass, $I=$ $10^{45} I_{45} \mathrm{~g} \mathrm{~cm}^{2}$ the moment of inertia of the NS. Then $V=$ $10^{7} V_{7} \mathrm{~cm} \mathrm{~s}^{-1}$ is the relative velocity between the NS and the wind. The mass capture rate of the NS $\dot{M}=10^{17} \dot{M}_{17} \mathrm{~g} \mathrm{~s}^{-1}$ is given by $\dot{M}=\pi R_{\mathrm{a}}^{2} \rho_{\infty} V$, where $R_{\mathrm{a}}=2 G M_{\mathrm{NS}} / V^{2}$ is the accretion radius and $\rho_{\infty}$ the density of the surrounding gas. When the spin period increases to the brake period $P_{\mathrm{br}}=$ $100 \mu_{31}^{16 / 21} \dot{M}_{17}^{-5 / 7} m^{-4 / 21} \mathrm{~s}$, the NS switches from subsonic propeller to accretor.

In the ejector stage, the transferred wind material is outside the light cylinder. The X-ray emission is generated from the rotational power of the NS. This will be discussed in Sect. 2.1.4. When the pressure of the ejected particles from the neutron star can no longer balance the ram pressure of the wind gas, the gas will penetrate into the light cylinder and interact with the star's magnetosphere, giving rise to X-ray emission. This propeller stage can be further divided into supersonic and subsonic stages (DP81). The corresponding X-ray luminosities are evaluated according to the work by Ikhsanov (2001).

Finally when the magnetic pressure is less than the ram pressure of the falling material and the material at the magnetospheric boundary is cool enough to trigger the interchange instabilities, the NS enters the accretor stage, and all the material captured by the NS is assumed to reach its surface. We then use $L_{\mathrm{X}}=\eta \dot{M} c^{2}$ with $\eta \simeq 0.1$ to calculate the X-ray luminosity.

The parameters of the natal NSs are set as follows. The initial spin periods $P$ and magnetic fields $B$ are chosen so that $\log P$ and $\log B$ are distributed normally with a mean of -2.3 and 12.5 , respectively, and a standard deviation of 0.3 .

\subsubsection{Magnetic CVs}

Muno et al. (2004a) suggest that the majority of the GC sources are IPs. To examine this idea, we include CVs in our calculations, and set $5 \%$ of them as IPs (Kube et al. 2003).

\subsubsection{LMXBs}

This type of system contains NSs or black holes (BHs) accreting from low-mass companions that overfill their Roche lobes. Most LMXBs are transients, with the majority of their time spent in quiescence with $L_{\mathrm{X}}<10^{34} \mathrm{erg} \mathrm{s}^{-1}$. The criterion suggested by van Paradijs (1996) is used to determine whether the X-ray source is persistent or a soft X-ray transient (SXT). BT04 took a semi-empirical approach to the X-ray luminosities of LMXB transients in quiescence. We have instead adopted the theoretical model of Menou et al. (1999) for quiescent SXTs, in which the inner part of the accretion flow is advection-dominated (ADAF, see Narayan et al. 1998, for a review), surrounded by an outer thin disk. For BH SXTs, the quiescent luminosities are

$L_{\mathrm{X}, \mathrm{q}}=g_{\mathrm{BH}} /\left(1+g_{\mathrm{BH}}\right) \times 10^{-5} \times \eta \dot{M} c^{2}$,

where $g_{\mathrm{BH}} \equiv \dot{M}_{\mathrm{ADAF}} / \dot{M}_{\mathrm{acc}} \simeq 0.5, \dot{M}_{\mathrm{ADAF}}$ is the rate at which mass is accreted via the ADAF, and $\dot{M}_{\text {acc }}$ the rate at which mass 
is accumulated in the outer thin disk. The accumulated mass will be accreted in the outbursts.

Menou et al. (1999) included the propeller effect to estimate the quiescent X-ray luminosities of NS transients

$L_{\mathrm{X}, \mathrm{q}}=g_{\mathrm{NS}} /\left(1+g_{\mathrm{NS}}\right) \times f_{\mathrm{acc}} \times \eta \dot{M} c^{2}$,

where $g_{\mathrm{NS}} \simeq 0.2$ and $f_{\mathrm{acc}}$ is the fraction of the total mass through the ADAF that reaches the NS surface.

Both normal star and white dwarf (WD) donors are considered in our simulations. Apart from core collapse SNe, BT04 suggests the formation of NSs via the accretion-induced collapse (AIC) of massive WDs. The EPS code in our simulation also includes this possibility: steady transfer of He-rich or C-rich material onto an ONe WD can lead to an AIC, leaving an NS remnant, when the WD masses exceed the Chandrasekhar mass limit.

\subsubsection{Rotation-powered pulsars}

In the ejector stage, the spin-down of NSs is governed by the canonical rotation-powered pulsar mechanism. The rotation power is spent to generate the magnetic dipole waves and accelerate particles. Young pulsars are X-ray emitters. There appears to be a strong correlation between the rate of rotational energy loss $\dot{E}$ and their X-ray luminosities $L_{\mathrm{X}}$ (Seward \& Wang 1988; Becker \& Trumper 1997; Saito 1998). The comprehensive investigation by Possenti et al. (2002) suggests that the X-ray luminosities in 2-10 keV band depend on $\dot{E}$ with the following relation

$L_{\mathrm{X}}=10^{-15.3}\left(\dot{E} / \mathrm{erg} \mathrm{s}^{-1}\right)^{1.34} \mathrm{erg} \mathrm{s}^{-1}$.

Since $\dot{E}$ decreases with time, rotation-powered pulsars can shine in X-rays with luminosities $\sim 10^{31}-10^{35} \mathrm{erg} \mathrm{s}^{-1}$ at ages of $\sim 10^{3}-10^{4}$ yr. The X-ray flux contains both pulsed nonthermal X-ray emission from the NS magnetosphere and unpulsed component from the synchrotron nebulae powered by the relativistic particles and magnetic fields ejected by the NS. Contribution from the NS cooling is most likely in the energy band $\leq 2 \mathrm{keV}$ and could be highly attenuated by the Galactic interstellar absorption.

\subsubsection{Massive stars with strong winds}

Massive stars with strong winds, like Wolf-Rayet stars, long have been known to be bright X-ray sources (Pallavicini et al. 1981). The X-ray emission is generally thought to be generated in the lower layers of the winds, where the plasma is heated in shocks arising from small-scale wind structures that grow out from line-driven wind instabilities (Feldmeier et al. 1997; Dessart \& Owocki 2003). Chelbowski \& Garmany (1991, hereafter CG91) found that the X-ray luminosities of both WolfRayet stars and $\mathrm{O}$ type stars can be fitted by the following relation,

$\log L_{\mathrm{X}}\left(\mathrm{erg} \mathrm{s}^{-1}\right)=34.32+0.34 \log \left(\dot{M}_{\mathrm{w}} v_{\infty}\right)$,

where $\dot{M}_{\mathrm{w}}$ is the wind mass loss rate in units of $\dot{M}_{\odot} \mathrm{yr}^{-1}$ and $v_{\infty}$ the terminal velocity of the mass outflows in units of $\mathrm{km} \mathrm{s}^{-1}$.
Massive binaries can add considerable X-rays due to collision of the winds from the two stars under specific conditions. First, this X-ray enhancement is significant only when the binary separation $a$ lies in the following range (CG91),

$1.1\left(R_{1}+R_{2}\right) \leq a \leq 5.5\left(R_{1}+R_{2}\right)$,

where $R$ is the stellar radius, and the subscripts 1 and 2 denote the primary and secondary stars, respectively. Second, Prilutskii \& Usov (1976) showed that the X-ray emission due to wind collision in binary systems is inefficient unless $\dot{M}_{2} v_{2} /\left(\dot{M}_{1} v_{1}+\dot{M}_{2} v_{2}\right) \geq 0.4$. If the above requirements are satisfied, we use the empirical relation of Portegies Zwart et al. (2002a) to calculate the X-ray luminosities for colliding winds,

$$
\begin{aligned}
L_{\mathrm{X}}= & 1.3 \times 10^{34}\left(\frac{\dot{M}_{1}}{10^{-5} M_{\odot} \mathrm{yr}^{-1}}\right)^{0.4} \\
& \times\left(\frac{v_{1}}{10^{3} \mathrm{~km} \mathrm{~s}^{-1}}\right)^{-0.65}\left(\frac{a}{R_{\odot}}\right)^{-0.2} \mathrm{erg} \mathrm{s}^{-1} .
\end{aligned}
$$

\subsection{X-ray band and luminosities}

Wang et al. (2002) survey revealed $\sim 1000$ discrete sources, most of which were detected in the relative hard energy range of $2-10 \mathrm{keV}$. Less than 20 of these sources are previously known (bright X-ray binaries). They suggest that half of these hard X-ray sources could be background active galactic nuclei (AGN). Considering the steep density profile in the Galactic disk, at least 100 sources with luminosities $10^{33}-10^{35} \mathrm{erg} \mathrm{s}^{-1}$ (Pfahl et al. 2002) are in the GCR. Among the 2357 point sources presented by Muno et al. (2003), 281 sources were detected below $1.5 \mathrm{keV}$ and are believed to be mainly in the foreground of the GC, and $\sim 100$ sources are suggested to be background AGN. The surface density of the remaining sources, and their absorption column toward the GC, in agreement with those in infrared surveys toward Sgr A*, demonstrate that these X-ray sources trace the general stellar population at the GCR (Muno et al. 2003). A tiny fraction of the remaining sources would be contaminated by the forground objects because of the steep density profile of the Galactic disk. In fact, the ratio of the foreground and the GCR sources $(8.5 \mathrm{kpc} \times 1) /(400 \mathrm{pc} \times 1000) \approx 0.02$ (see Sect. 2.4 for the GCR stellar density prescription), is negligible. By using the typical spectral model obtained from those relatively bright sources in this sample, Muno et al. (2003) infer the 2.0-8.0 keV luminosity range of $10^{30} \sim 10^{33} \mathrm{erg} \mathrm{s}^{-1}$ for the GC sources. The luminosities estimated for some of the models described in Sect. 2.1 are bolometric, and have to be converted into the $2-10 \mathrm{keV}$ luminosities to be compared with observations.

The WNSs often exhibit non-thermal spectra that can be described with a $\Gamma \sim 0$ power law below $10 \mathrm{keV}$ (e.g. Campana et al. 2001), we thus adopt their $2-10 \mathrm{keV}$ luminosities to be half the calculated bolometric luminosities. Magnetized CVs, especially IPs, usually exhibit hard thermal spectra $(k T \geq$ $10 \mathrm{keV}$ ) (e.g. Ezuka \& Ishida 1999). We also take their bolometric correction factor to be 0.5 . The theoretical model of Menou et al. (1999) of quiescent X-ray transients is established for comparison with observational luminosities in $0.5-10 \mathrm{keV}$, 
we multiply them by a factor of 0.5 to estimate the luminosities in $2-10 \mathrm{keV}$. The X-ray luminosities determined by Eq. (7) for rotation-powered pulsars are appropriately in $2-10 \mathrm{keV}$, so no correction is needed. CG91 obtained the empirical relation (Eq. (8)) for massive stars with strong winds from the data of the Einstein observatory (in $0.5-3.5 \mathrm{keV}$ ). For comparison, Portegies Zwart et al. (2002a) calculated the luminosities in the $0.5-3.5 \mathrm{keV}$ band (Eq. (10)) based on the archival Chandra X-ray observations. Taking account of their soft spectra ( $k T \sim 1-3 \mathrm{keV}$, Portegies Zwart et al. 2002a), we choose the correction factor to be 0.1 for their $2-10 \mathrm{keV}$ luminosities.

The X-ray emission is generally assumed to be isotropic. However, for disk accreting sources, the geometric effect may affect the apparent luminosity distributions (Zhang 2005). Quantitatively, the intrinsic luminosity is reduced by a factor of $\cos \theta(1+2 \cos \theta) / 3$, where $\theta$ is the inclination angle of the accretion disk; the factor of $\cos \theta$ is due to the area-projection effect, and the factor of $(1+2 \cos \theta) / 3$ is due to the limbdarkening effect (Netzer 1987). The probability of seeing an accretion disk at an inclination angle $\theta$ is proportional to $\sin \theta$ if accretion disks are assumed to be oriented randomly in the sky. Therefore, the convolution between $f(x)=\left(1-x^{2}\right)^{1 / 2}[1+$ $2\left(1-x^{2}\right)^{1 / 2}$ ] (where $x=\sin \theta$ is uniformly distributed between 0 and 1) and a given intrinsic luminosity distribution produces the observed distribution (see e.g. Figs. 2, 5, and 6). Thus, we take this inclination effect into account for Roche lobe overflow (RLOF) X-ray binaries (i.e. IP and LMXBs).

\subsection{Star formation and IMF in GCR}

As noted by Muno et al. (2004a), it is still a matter of debate as to whether the star formation is continuous or episodic and whether it occurs only in localized regions or is relatively uniform throughout the GC. By modeling the evolution of the population of luminous infrared stars, Figer et al. (2004) suggests that the star formation is probably continuous over the last $\sim 10 \mathrm{Gyr}$, as we do in this paper. The Kroupa et al. (1993) IMF adopted in this work has a slope of -2.7 when $M>1.0 M_{\odot}$, derived from the stellar distribution towards both Galactic poles, as well as from the distribution of stars within $5.2 \mathrm{pc}$ from the Sun. However, the molecular clouds in the GCR have higher densities and temperatures than those in the disk due to the tidal forces. This environment might favor the formation of massive stars (Morris 1993). The Hubble Space Telescope observation of Arches Cluster (which is within the Wang et al. field) suggested a fairly flat IMF with a slope of $\sim-1.7--1.9$ (Figer et al. 1999; Figer 2005; see also Stolte et al. 2002). Portegies Zwart et al. (2002b), however, show that the observed characteristics (unusually flat mass function and overabundance of massive stars) of the Arches cluster are consistent with a perfectly normal IMF. The observed anomalies are then caused by a combination of observational selection effects and the dynamical evolution of the cluster. Considering the lack of knowledge of the star formation history in GCR, our assumptions about star formation and IMF in the GCR are not incompatible with recent observational and theoretical works.
Table 1. Model parameters.

\begin{tabular}{ccccc}
\hline \hline Model & $\alpha_{\mathrm{CE}}$ & $\beta$ & $\sigma$ & tides \\
\hline $\mathrm{A}$ & 1.0 & 1.0 & 265 & $\mathrm{OFF}$ \\
$\mathrm{B}$ & 0.5 & 1.0 & 265 & $\mathrm{OFF}$ \\
$\mathrm{C}$ & 1.0 & 4.0 & 265 & $\mathrm{OFF}$ \\
$\mathrm{D}$ & 1.0 & 0.25 & 265 & $\mathrm{OFF}$ \\
$\mathrm{E}$ & 1.0 & 1.0 & 265 & $\mathrm{ON}$ \\
$\mathrm{F}$ & 1.0 & 1.0 & 190 & $\mathrm{OFF}$ \\
$\mathrm{G}$ & 1.0 & 1.0 & 190 & $\mathrm{ON}$ \\
\hline
\end{tabular}

\subsection{Expected numbers}

The expected numbers for each class of X-ray sources in the GCR are related to the the ratio of the star population in the GCR and in the total disk. The size of Wang et al. survey field is $2^{\circ} \times 0.8^{\circ}$. Supposing the space density of stars in the Galactic disk to be $n(R, z) \propto \exp \left(-R / R_{0}\right) \exp \left(-|z| / z_{0}\right)$ and integrating $n(R, z)$ over $1.6 \mathrm{deg}^{2}$ through the GCR, Pfahl et al. (2002) found that the field contains $\leq 1 \%$ of the total Galactic disk population. Alternatively, we can make an order of magnitude estimate of this ratio. The Wang et al. survey field encompasses a physical area of $300 \mathrm{pc}$ by $120 \mathrm{pc}$ at a distance of $8.5 \mathrm{kpc}$. Assuming that all of the point sources are within the nuclear bulge (which is about $300 \mathrm{pc}$ across in the radial direction; Mezger et al. 1996) and the average stellar density is $100 M_{\odot} \mathrm{pc}^{-3}$ (which is 1000 times the one in the local neighborhood, Binney \& Merrifield 1998), the Wang et al. field contains a stellar mass of $300 \times 120 \times 300 \times 100=10^{9} M_{\odot}$. Thus, this field contains $1 \%$ of the total Galactic stellar mass, which is $\sim 10^{11} M_{\odot}$. With EPS calculations, we try to search various types of X-ray sources (described in Sect. 2) that can be detected in the Wang et al. (2002) and Muno et al. (2003) surveys. Once we get the total number $N_{\mathrm{Gal}}$ of a specific type of sources in the Galactic disk, then $\sim N_{\mathrm{Gal}} / 100$ and $\sim N_{\mathrm{Gal}} / 100 / 4$ sources of this population are expected to be found in the Wang et al. and the Muno et al. surveys, respectively, since the latter field contains 4 times less stars than the former (BT04). Note that those sources found in these two surveys have different luminosity ranges.

\section{Results}

We adopt a variety of models (see Table 1), each with different assumptions for the parameters that govern the evolutions in the calculations. Tables 2 and 3 summarize the calculated numbers of various classes of X-ray sources that contributed to the Wang et al. and the Muno et al. surveys, respectively. The expected numbers after correcting for the inclination effect are also listed.

The luminosity distributions of WNSs are shown in Fig. 1. The solid and dashed lines represent the calculated results with and without the propeller effect considered, respectively. The latter distribution implies that there are about 12 and 16 WNSs (for Models A and D, respectively) in the Wang et al. field. PRP02 predicted $\sim 250$ WNSs in the solid angle of the Wang et al field for their standard kick model, and $\sim 12-100$ of them could be detected in the survey. This is compatible with our 
Table 2. The expected number of X-ray sources in the Wang et al. survey with $L_{\mathrm{X}, 2-10 \mathrm{keV}}=10^{33}-10^{35} \mathrm{erg} \mathrm{s}^{-1}$. In parentheses we list the expected numbers of X-ray binaries with WD donors. The expected numbers after correcting for the inclination effect are listed in the last column.

\begin{tabular}{cccccccc}
\hline \hline Model & WNSs & NS LMXBs & BH LMXBs & IPs & Pulsars & TOTAL & TOTAL-inc \\
\hline $\mathrm{A}$ & $<1$ & $3(0)$ & $<1(0)$ & 1 & 9 & 13 & 13 \\
$\mathrm{~B}$ & $<1$ & $<1(0)$ & $<1(0)$ & 1 & 9 & 9 & 9 \\
$\mathrm{C}$ & $<1$ & $4(0)$ & $<1(0)$ & 1 & 9 & 14 & 14 \\
$\mathrm{D}$ & $<1$ & $<1(0)$ & $<1(0)$ & 2 & 9 & 11 & 11 \\
$\mathrm{E}$ & $<1$ & $2(0)$ & $1(0)$ & 1 & 9 & 13 & 12 \\
$\mathrm{~F}$ & $<1$ & $23(0)$ & $<1(0)$ & 1 & 9 & 33 & 30 \\
$\mathrm{G}$ & $<1$ & $5(0)$ & $1(0)$ & 1 & 9 & 16 & 14 \\
\hline
\end{tabular}

Table 3. The expected numbers of X-ray sources in the Muno et al. survey with $L_{\mathrm{X}, 2-10 \mathrm{keV}}=10^{30}-10^{33} \mathrm{erg} \mathrm{s}^{-1}$. In parentheses we list the expected number of X-ray binaries with WD donors. The expected numbers after correcting for the inclination effect are listed in the last column.

\begin{tabular}{cccccccc}
\hline \hline Model & IPs & NS LMXBs & BH LMXBs & Pulsars & Massive Stars & TOTAL & TOTAL-inc \\
\hline A & 165 & $648(646)$ & $132(0)$ & 28 & 72 & 1045 & 912 \\
B & 169 & $195(194)$ & $7(0)$ & 28 & 71 & 470 & 405 \\
C & 163 & $642(641)$ & $130(0)$ & 28 & 72 & 1035 & 905 \\
D & 176 & $935(934)$ & $158(0)$ & 28 & 72 & 1369 & 1202 \\
E & 706 & $1142(1141)$ & $270(0)$ & 28 & 72 & 2218 & 1963 \\
F & 165 & $694(693)$ & $128(0)$ & 28 & 72 & 1087 & 961 \\
G & 706 & $1242(1240)$ & $497(0)$ & 28 & 71 & 2544 & 2248 \\
\hline
\end{tabular}

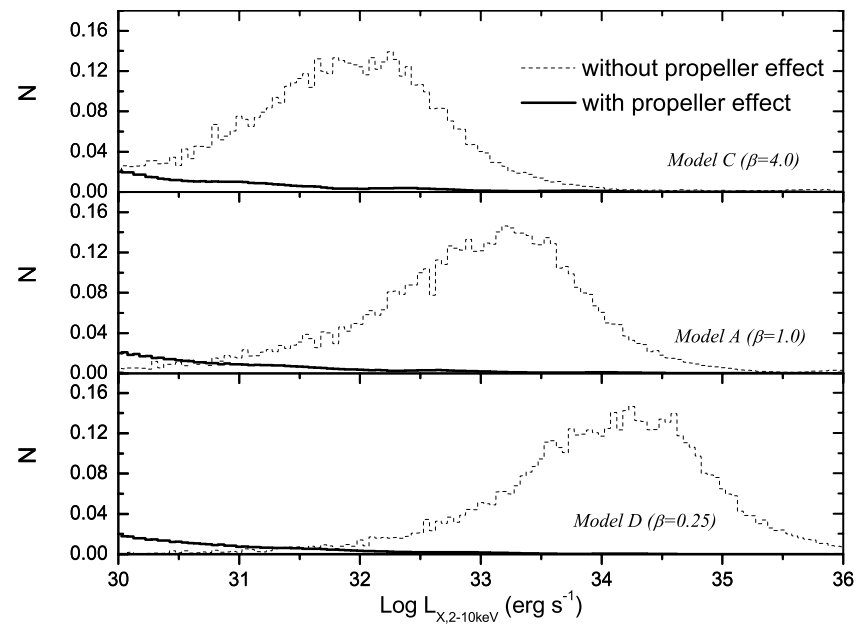

Fig. 1. The X-ray luminosity distributions for WNSs with and without considering the propeller effect. Three models C, A, and D with decreasing wind velocity are shown from top to bottom.

result, as we get 25 WNSs in Model F, in which the SN kick model is same as the standard model of PRP02. The figure also reveals that with decreasing wind velocities the WNS luminosities increase without considering the propeller effect, as expected with the Bondi-Hoyle mechanism. However, when we take account of the propeller effect, the expected number of WNSs that can be detected in the Wang et al. survey is less than one, indicating that mass accretion may be highly ineffcient during most part of the WNS stage.

Figure 2 shows the luminosity distributions of various classes of the RLOF X-ray binaries expected in the Muno et al. field. The thin solid lines, dashed lines, and thick solid lines are for IPs, NS LMXBs and BH LMXBs respectively. In this figure

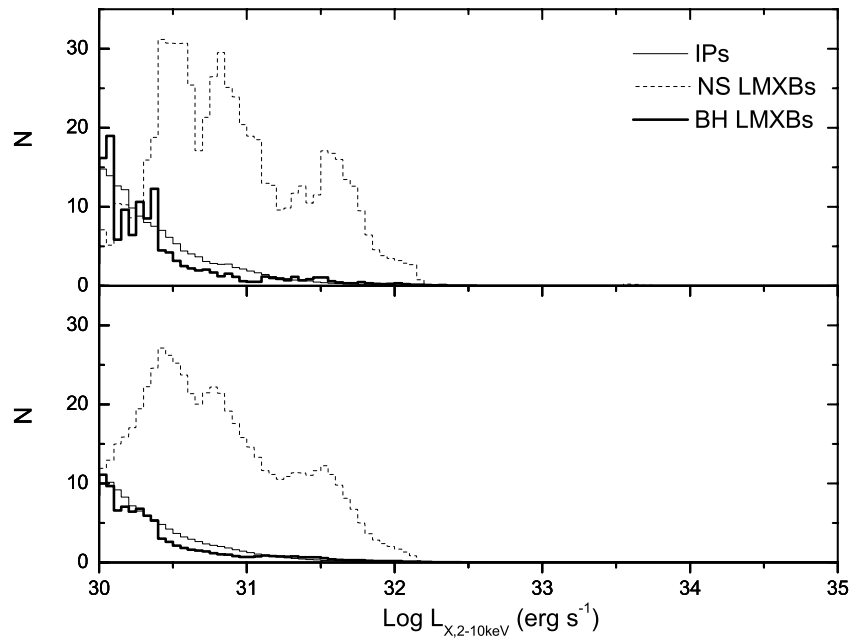

Fig. 2. The X-ray luminosity distributions for Roche-lobe overflow systems including IPs (thin solid lines), NS LMXBs (dashed lines), and BH LMXBs (thick solid lines). The figure is plotted only for Model A, since the main features do not change much as the parameters change. Upper panel: intrinsic distributions. Lower panel: distributions after correcting for accretion disk inclination effect.

we can see that NS LMXBs in quiescence, generally brighter than BH LMXBs, account for a considerable fraction of the sources in the Wang et al. survey (30 out of $\sim 100$ for model F, see Table 2); NS LMXBs with WD donor stars contribure a significant fraction of the sources in Muno et al. field. Most of the NS-WD LMXBs are ultracompact, with orbital periods less than $2 \mathrm{~h}$. Their numbers (see Table 3 ) are roughly in line with those (291) in BT04. Our simulations predict hundreds of IPs in the GCR, somewhat less than Muno et al. (2004a)'s estimate $(\sim 1000)$. 
The CE efficiency parameter $\alpha_{\mathrm{CE}}$ plays an important role in the evolution of close binaries. To see the dependence of the results on $\alpha_{\mathrm{CE}}$, we can compare the results of Model A $\left(\alpha_{\mathrm{CE}}=1.0\right)$ with those of Model B $\left(\alpha_{\mathrm{CE}}=0.5\right)$ in Table 3. The parameter $\alpha_{\mathrm{CE}}$ has two contrary effects on the evolution of close binaries. On one hand, the high value of $\alpha_{\mathrm{CE}}$ results in relatively wide binaries after the $\mathrm{CE}$ phase, so that the subsequent RLOF mass transfer might not be able to take place. On the other hand, for the ultracompact X-ray binaries, a high value of $\alpha$ can prevent coalescence during the previous CE phase, significantly increasing the formation rate of these systems. The overall influence is that larger $\alpha$ leads to more LMXBs, especially LMXBs with a WD donor, because these binaries have experienced CE evolution at least twice.

Both IP and LMXB numbers increase significantly when the tidal spin-orbit coupling is considered in Models $\mathrm{E}$ and $\mathrm{G}$. When the primary star ascends to the giant branch, its expansion causes a reduction of the orbital separation through the spin-orbit interaction, while wind mass loss from the evolved donor star causes the orbit to widen. The competition between the wind loss and the tidal interactions will determine the subsequent orbital evolution (Tauris \& Savonije 2000; Tauris 2001). As a typical example of CV formation in Model $\mathrm{E}$ (TIDE ON) let us consider binary stars of masses 2.9 and $0.4 M_{\odot}$ in an orbit with an initial separation of $484 R_{\odot}$. About 0.5 Gyr later, the more massive star evolves to the AGB stage when the separation is $488 R_{\odot}$. In the following $3 \mathrm{Myr}$ evolution, the tidal spin-orbit interaction dominates the orbital evolution and the binary separation decreases by $30 \%$ to $355 R_{\odot}$ when the primary star begins to fill its Roche lobe. A CE forms and a $\mathrm{CO}$ white dwarf separated from the $0.4 M_{\odot}$ main sequence (MS) star by $2 R_{\odot}$ emerges. When the separation has fallen to $1.1 R_{\odot}$ (due to tidal interaction) $70 \mathrm{Myr}$ later, the secondary star fills its Roche lobe and the stage of CV evolution begins. In contrast, the same system in Model A (TIDE OFF) has an ever increasing orbital separation (from 484 to $500 R_{\odot}$, owing to the wind mass loss effect) before the primary star fills its Roche lobe. The CE evolution leads to a CO WD-MS star binary with a separation of $3 R_{\odot}$. The secondary star cannot fill its Roche lobe within $12 \mathrm{Gyr}$ because the timescale for either its nuclear expansion or the orbital shrinkage due to gravitational radiation is too long.

In Fig. 3 we show the X-ray luminosity distributions of rotation-powered pulsars. There are in total $\sim 28$ and $\sim 9$ pulsars predicted in the Muno et al. and the Wang et al. surveys respectively. The numbers of rotation-powered pulsars rely only on the core-collapse SN rate and their X-ray lifetime, both independent of the parameters in Table 1.

Figure 4 shows the X-ray luminosity distributions of massive stars with strong winds. Only a small fraction of them have colliding winds and thus are more luminous. Their luminosities increase with decreasing wind velocities. The reason is that the $\mathrm{X}$-ray luminosity from colliding winds is proportional to the product of the emissivity per unit volume, $n^{2} \Lambda$, and both the density $n$ and emission rate $\Lambda$ are anticorrelated with the wind velocity $v_{\mathrm{W}}$. We obtain $\sim 70$ massive stars in the Muno et al. survey. The number hardly changes with the model parameters.

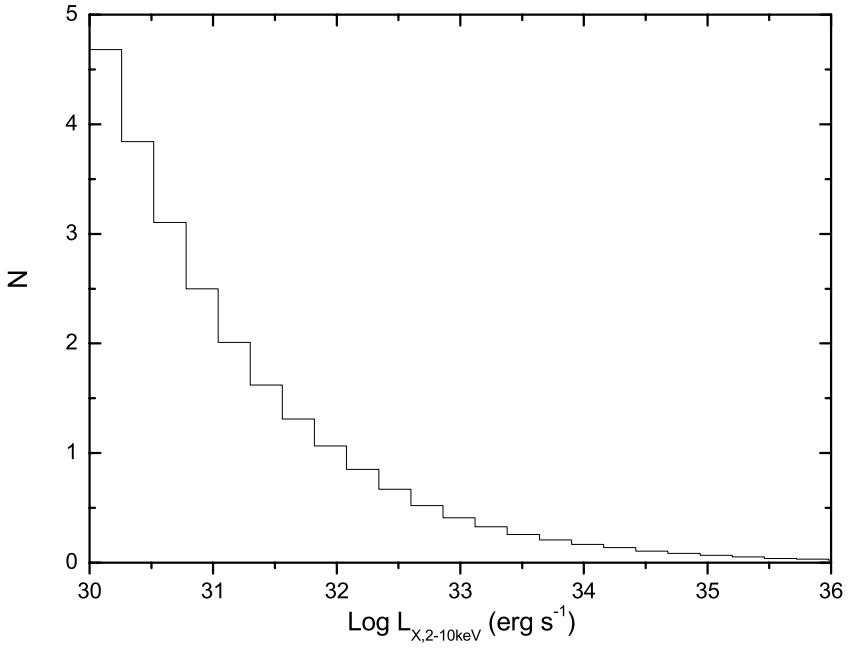

Fig. 3. The X-ray luminosity distributions for rotation-powered pulsars. This figure is plotted for Model A. Distributions for other models show few changes.

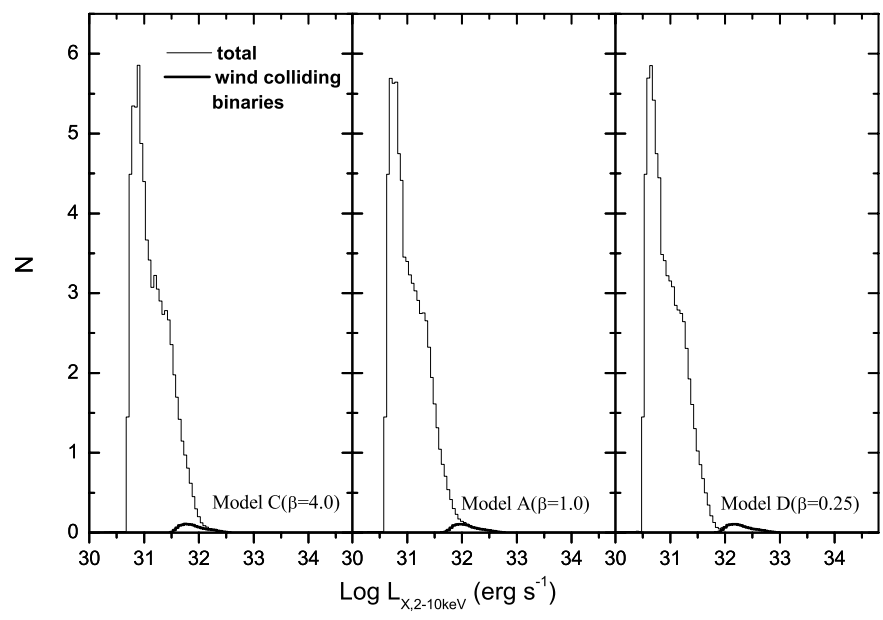

Fig. 4. The X-ray luminosity distributions for massive stars with strong winds (dashed lines). Distributions for wind-colliding binaries are highlighted with thick lines. Three Models C, A, and D with decreasing wind velocity are shown from left to right.

Figures 5 and 6 show the X-ray luminosity distributions of the total X-ray sources for different models. All models (especially models $E$ and $G$ with TIDE $O N$ ) seems to produce X-ray sources with reasonable numbers in the Muno et al. field, but less than half the objects in the Wang et al. field are accounted for. In Muno et al. (2003) the luminosity distribution peaks around $2 \times 10^{31} \mathrm{erg} \mathrm{s}^{-1}$, nearly one order of magnitude higher than in our work. The reasons for this discrepancy can be addressed briefly as follows. First the calculated mass transfer rates are long-term, averaged ones. They may not be directly compared with observed, instantaneous X-ray luminosities. Second, observational selection effects may favor the relatively more luminous X-ray sources to be identified. Third, some potentially important effects (such as the effect of X-ray irradiation on the secondary) have not been included in our binary calculations, which could considerably alter the evolution of these systems and increase the mass accretion rates (see also Podsiadlowski et al. 2002). 


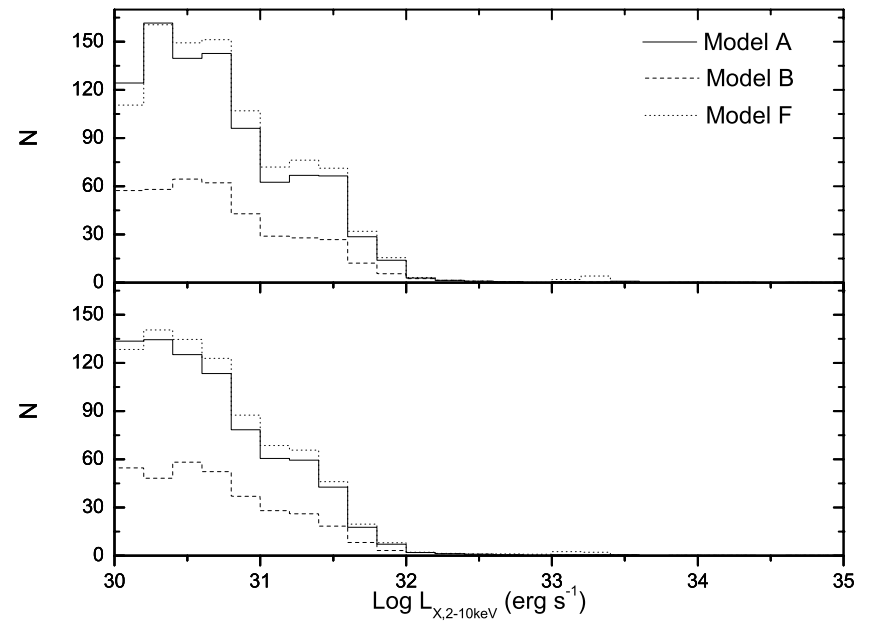

Fig. 5. The total number of X-ray sources as a function of the X-ray luminosity. Three models, A (solid lines), B (dashed lines), and F (dotted lines), in which no tidal effect was considered, are shown. Upper panel: intrinsic distributions. Lower panel: distributions after correcting for the accretion disk inclination effect.

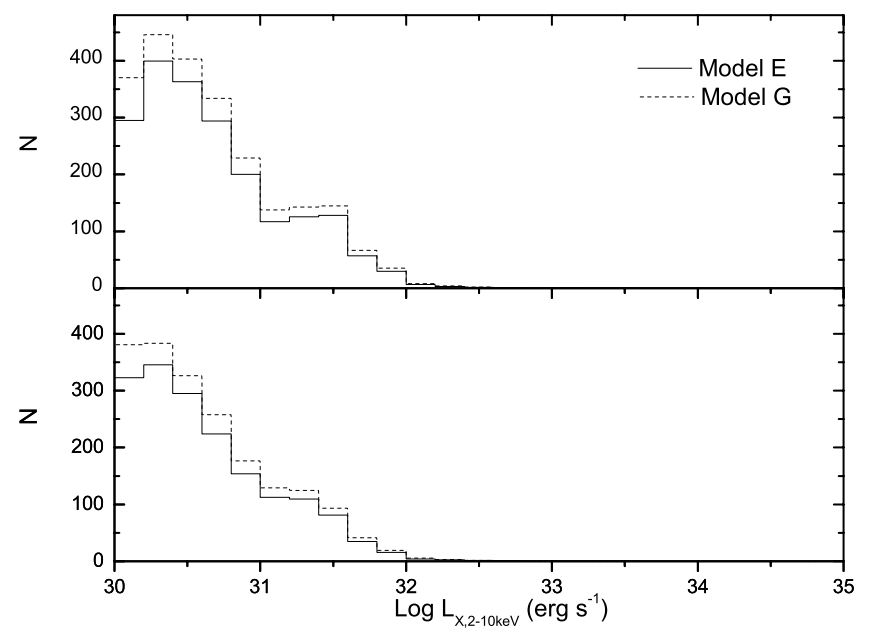

Fig. 6. Same as Fig. 5. The two models, E (solid lines) and G (dashed lines), in which tidal effect was considered are shown here. Upper panel: intrinsic distributions. Lower panel: distributions after correcting for the accretion disk inclination effect.

We also calculate the numbers of bright X-ray sources in GCR. The outburst correction factor is chosen to be 0.1 and 1 for the short- and long-period LMXB transient systems, respectively (see BT04 for details). We assume the bolometric correction factor is 0.1 and the duty cycle is $1 \%$. For Model A, $23 \mathrm{X}$-ray sources brighter than $10^{36} \mathrm{erg} \mathrm{s}^{-1}$ are found in the Muno et al. field, including 16 transient LMXBs in outburst and 7 persistent LMXBs. Only a few bright transients are detected in the Muno et al. survey. For the Wang et al. field, we predict 92 bright point sources, whereas $\leq 20$ bright sources were detected in Wang et al. (2002). Like BT04, our simulation seems to lead to an overproduction of the bright systems. However, for model B, the expected numbers of bright sources in the Muno et al. and Wang et al. surveys are reduced to 9 and 36 , respectively.
We calculate the point source contribution to the X-ray emission that has previously been ascribed to diffuse emission (Koyama et al. 1996; Sidoli \& Mereghetti 1999) in the GCR. For various models, the X-ray point sources $\left(\leq 10^{35} \mathrm{erg} \mathrm{s}^{-1}\right)$ produce a mean surface brightness of 1.3-2.6 $\times$ $10^{-14} \mathrm{erg} \mathrm{cm}^{-2} \mathrm{~s}^{-1} \operatorname{arcmin}^{-2}$. This is 3\%-9\% of that of the diffuse emission from the inner regions of the Galaxy derived by Sidoli \& Mereghetti (1999) and by Koyama et al. (1996). Our result is compatible with the observational result (10\%) of Muno et al. (2003).

\section{Discussion and conclusions}

Using the EPS method, we have investigated the nature of the faint X-ray sources in the GCR. Our simulated objects include WNSs, magnetic CVs, LMXBs, rotation-powered pulsars, and massive stars with strong winds. The main results are summarized in Tables 2 and 3, with $\sim 10-30$ and $\sim 1000-2000$ sources expected to be detected in the Wang et al. and Muno et al. surveys, respectively. For the Wang et al. survey, we found that a considerable fraction of the discrete sources may be rotationpowered pulsars and NS LMXB transients in quiescence, while the WNSs proposed by PRP02 make a negligible contribution due to the propeller effect. Note that we have not considered $\mathrm{Be} / \mathrm{X}$-ray binaries in which the NS evolution may be quite different from investigated here. Recent near infrared imaging of the X-ray sources in the GCR shows that the color distribution of the identified candidate counterparts of some of the X-ray sources is redder than expected for WNS systems, but is consistent with later-type stars (Bandyopadhyay et al. 2005). For the Muno et al. field, IPs present a minor contribution, and the majority of the X-ray sources seem to be NS LMXB transients with WD donors. The latter result is consistent with BT04.

Our calculations also suggest that some of the point sources detected by the Muno et al. survey may be massive stars with strong winds. Radio emission from these winds should be detectable at centimeter wavelengths (Panagia \& Felli 1975; Wright \& Barlow 1975). We propose that a systematic radio observing campaign be undertaken to search for the stellar counterparts in the GCR surveyed by Muno et al. (some efforts have been made, e.g. by Lang et al. 2005, most recently). Moreover, radio observations could reveal the existence of jets, which are common in X-ray binaries.

Sakano et al. (2005) have recently reported the discovery in the GCR of two unusual X-ray transients XMM J174457-2850.3 and XMM J174544-2913.0 with flux variations in excess of a factor of 100 during roughly a year and peak X-ray luminosities of $\sim 5 \times 10^{34} \mathrm{erg} \mathrm{s}^{-1}$ and with peculiar spectral features. Since no known classes of sources can explain all their characteristics well, these authors argue that these two sources may represent a new type of source with different properties from those we have known. This also implies that what is happening in the GCR is much more complicated than we have already learnt.

Acknowledgements. We would like to thank Jarrod R. Hurley for kindly providing us his SSE and BSE codes and for valuable conversations. We thank Z.-R. Wang, Y. Chen, Z.-W. Han, and M. Li for 
useful discussions. We are grateful to the anonymous referee for constructive suggestions for improving the manuscript. This work was supported by the National Natural Science Foundation of China under grant 10573010 and the Ministry of Science and Technology of China under grant NKBRSF G19990754.

\section{References}

Bandyopadhyay, R. M., Miller-Jones, J. C. A., Blundell, K. M., et al. 2004, in Proc. of Interacting Binaries: Accretion, Evolution, and Outcomes, ed. L. A. Antonelli et al. (Cefalu, Sicily: AIP) [arXiv:astro-ph/0501327]

Becker, W., \& Trumper, J. 1997, A\&A, 326, 682

Belczynski, K., \& Taam, R. E. 2004, ApJ, 616, 1159 (BT04)

Bondi, H., \& Hoyle, F. 1944, MNRAS, 104, 273

Binney, J., \& Merrifield, M. 1998, Galaxy Astronomy (Princeton University Press)

Campana, S., Gastaldello, F., Stella, L., et al. 2001, ApJ, 561, 924

Chelbowski, T., \& Garmany, C. D. 1991, ApJ, 368, 241 (CG91)

Davies, R. E., \& Pringle, J. E. 1981, MNRAS, 196, 209 (DP81)

Dessart, L., \& Owocki, S. P. 2003, A\&A, 406, 1

Ezuka, H., \& Ishida, M. 1999, ApJS, 120, 277

Feldmeier, A., Puls, J., \& Pauldrach, A. W. A. 1997, A\&A, 322, 878

Figer, D. F. 2005, in the IMF@50, Conf. Proc., to be published [arXiv:astro-ph/0409415]

Figer, D. F., Kim, S. S., Morris, M., et al. 1999, ApJ, 525, 750

Figer, D. F., Michael, R. R., Kim, S. S., Morris, M., \& Serabyn, E. 2004, ApJ, 601, 319

Hansen, B. M. S., \& Phinney, E. S. 1997, MNRAS, 291, 569

Hobbs, G., Lorimer, D. R., Lyne, A. G., \& Kramer, M. 2005, MNRAS, 360, 963

Hurley, J. R., Pols, O. R., \& Tout, C. A. 2000, MNRAS, 315, 543

Hurley, J. R., Tout, C. A., \& Pols, O. R. 2002, MNRAS, 329, 897

Ikhsanov, N. R. 2001, A\&A, 375, 944

Ikhsanov, N. R. 2002, A\&A, 381, 61

Koyama, K., Maeda, Y., Sonobe, T., et al. 1996, PASJ, 48, 249

Kroupa, P., Tout, C. A., \& Gilmore, G. 1993, MNRAS, 262, 545

Kube, J., Gansicke, B. T., Euchner, F., \& Hoffmann, B. 2003, A\&A, 404, 1159

Lang, C. C., Johnson, K. E., Goss, W. M., \& Rodriguez, L. F. 2005, AJ, in press [arXiv: astro-ph/0508178]

Lipunov, V. M. 1992, Astrophysics of neutron stars (Heidelberg: Springer-Verlag)
Menou, K., Esin, A. A., Narayan, R., et al. 1999, ApJ, 520, 276

Mezger, P. G., Duschl, W. J., \& Zylka, R. 1996, A\&AR, 7, 289

Morris, M. 1993, ApJ, 408, 496

Muno, M. P., Baganoff, F. K., Bautz, M. W., et al. 2003, ApJ, 589, 225

Muno, M. P., Arabadjis, J. S., Baganoff, F. K., et al. 2004a, ApJ, 613, 1179

Muno, M. P., Baganoff, F. K., Bautz, M. W., et al. 2004b, ApJ, 613, 326

Narayan, R., Mahadevan, R., \& Quataert, E. 1998, in The Theory of Black Hole Accretion Discs, ed. M. A. Abramowicz, G. Bjornsson, \& J. E. Pringle (Cambridge: Cambridge University Press) [arXiv: astro-ph/9803141]

Netzer, H. 1987, MNRAS, 225, 55

Pallavicini, R., Golub, L., Rosner, R., et al. 1981, ApJ, 248, 279

Panagia, N., \& Felli, M. 1975, A\&A, 39, 1

Pfahl, E., Rappaport, S., \& Podsiadlowski, P. 2002, ApJ, 571, L37 (PRP02)

Podsiadlowski, P., Rappaport, S., \& Pfahl, E. D. 2002, ApJ, 565, 1107

Popov, S. B. 2004, A\&A, 418, 699

Possenti, A., Cerutti, R. Colpi, M., \& Mereghetti, S. 2002, A\&A, 387, 993

Portegies Zwart, S. F., Pooley, D., \& Lewin, W. H. G. 2002a, ApJ, 574,762

Portegies Zwart, S. F., Makino, J., McMillan, S. L. W., \& Hut, P. 2002b, ApJ, 565, 265

Prilutskii, O. F., \& Usov, V. V. 1976, Astr. Zh., 53, 6

Sakano, M., Warwick, R. S., Decourchelle, A., \& Wang, Q. D. 2005, MNRAS, 357, 1211

Saito, Y. 1998, Ph.D. Thesis, Univ. of Tokyo

Seward, F. D., \& Wang, Z. 1988, ApJ, 332, 199

Sidoli, L., \& Mereghetti, S. 1999, A\&A, 349, L49

Stolte, A., Grebel, E. K., Brandner, W., \& Figer, D. F. 2002, A\&A, 394,459

Tauris, T. M., \& Savonije, G. J. 2000, Proc. of the NATO ASI, The Neutron Star - Black Hole Connection [arXiv: astro-ph/0001014]

Tauris, T. M. 2001, Evolution of Binary and Multiple Star Systems, ASP Conf. Ser., ed. P. Podsiadlowski et al. [arXiv: astro-ph/0012077]

van Paradijs, J. 1996, ApJ, 464, L139

Wang, Q. D., Gotthelf, E. V., \& Lang, C. C. 2002, Nature, 415, 148

Willems, B., \& Kolb, U. 2003, MNRAS, 343, 949

Wright, A. E., \& Barlow, M. J. 1975, MNRAS, 170, 41

Zhang, S. N. 2005, ApJ, 618, L79 